\title{
Collaborative Group Label Work System based on Web Technology
}

\author{
Syuuya Tanaka*, Motoki Miura *
}

\begin{abstract}
Group label work (ex. KJ method) has been performed using papers such as sticky notes and a large paper sheet. However, using sticky notes has a drawback in terms of editing, managing and storage of working outcomes, and it is also difficult to digitize. Similar softwares and tools that help group label work have been proposed, but most of the tools depend on the specific platforms/operating systems. Therefore, we have developed a collaborative group label work system based on standard web technologies. By adopting the standard web technologies, the system works on multiple heterogeneous devices including PCs, tablets, and smart phones as a web application. In this paper, we describe the design and implementation of the method. We also compared the proposed method and the system with conventional paper-based group label work in terms of creativity and efficiency.
\end{abstract}

Keywords: groupware, idea, generation, tabletop, web application

\section{Introduction}

The KJ method[1] is an idea generation method devised by Jiro Kawakita. The KJ method has been widely used alongside brainstorming. The purpose of the KJ method is to find new ideas and perspectives from combining a plurality of information such as ideas, thoughts, and data. We explain the procedure of the KJ method as follows. First, participants generate ideas based on a given theme through brainstorming, and write down the ideas on paper la-bels. During the brainstorming, the participants are expected not to criticize other's opinion. Rather than criticism, the participants are expected to externalize their ideas aggressively. After the participants generated the ideas, they move to an idea organizing phase. In the idea organizing phase, the participants browse the collected ideas and find similar contents from the idea labels by their intuition. The similar ideas are gathered into a group. The group (sometimes, it is called as "island") is also labeled by appropriate title by the participants. By repeating the idea organizing phases, the participants can construct a structure of an affinity diagram.

After constructing the structure, the participants expand the structure of the grouped label, and organize an affinity diagram. The process of making an affinity diagram is also

\footnotetext{
* Kyushu Institute of Technology, Fukuoka, Japan
} 
called "type-A illustration." The affinity diagram can represent the interrelationships between groups (islands) and inner labels by their location and distance. Finally, the participants write out the summary of the affinity diagram by considering the association of the groups. The process is also called "type-B narrative."

In the KJ method, participants generally use a large paper for a base sheet and sticky notes for labels. The paper and sticky notes are intuitive and efficient for collaborative group work. However, when it comes to re-writing and modifying the content such as label and group title, the paper-based interface is not efficient. Also, in terms of the stability and preservation of the affinity diagrams, the paper-based interface is not suitable. Because the sticky notes tend to peel off. Capturing the whole affinity diagrams could be possible by taking photos, but the captured image is not suitable for re-editing. Miura et al. [2] proposed a digitizing method by using digital pen. This approach can solve both the stability and preservation issues. However, the participants have to perform additional operations to make digitized version of affinity diagrams.

In recent years, the spread of Internet accessible mobile devices such as smartphones and tablets, users can easily publicize and share their opinions and thoughts from any place at any time. Especially a tablet usually have large-sized display. Even though each display size of the tablet is not so large for performing KJ method, it is possible to organize pseudo large display environment if multiple tablets are arranged.

\section{Purpose}

In this paper, we propose a collaborative group label work system by adopting multiple tablets. By combining multiple tablets, the participants can obtain wider area of the shared workspace.

Many researches and systems have been performed to form a large-scale interactive tabletop display. There researches can be categorized into two groups. One is develop-ing a special hardware to enable an interactive surface, including multi-touch and useridentification[3, 4]. The other is to form a large-scale interactive surface by adopting conventional projectors and/or LCD panels, with attaching additional sensors and cameras for realizing interactive operations[5, 6]. Our approach is similar to the later group. But by adopting the standard web technologies, the proposed method is applicable for casual and ad-hoc collaboration since no special sensors and devices are necessary. The participants can prepare the shared workspace by bringing their own devices. The standard web tech-nology enables heterogeneous tablet devices to form the joined large shared workspace virtually.

\section{Method}

In this paper, we propose a collaborative group label work system with multiple heterogeneous tablets by utilizing standard web technologies. The merit of utilizing standard web technologies is that there are no special softwares installed in the tablets, beforehand the collaborative group work. Especially, WebSocket[7] is one of the key technologies of modern web application. Using WebSocket technology, a web server can send messages to the client connected at any time. The message can update the screen of the client. Also, the clients can send messages to the server for updating the shared model, as usual web requests, depending on the participants operations. Therefore, it 
is suitable for realizing real-time communication and collaboration[8] even with multiple tablet devices. By combining multiple tablets, the participants can obtain wider area of the shared workspace. The wider screen is appropriate for performing a group label work that handles multiple labels.

As a tablet, we consider a multi-touch enabled device such as iPad and Android OS tablets. The multi-touch capability is crucial for intuitive operations, especially for the group label work system. Because during KJ method, participants want to move multiple labels at a same time. Therefore, simultaneous moving of labels can be improve the usability of the system.

We assume the tablet device has installed a modern web browser, such as Mobile Safari, Google Chrome, and so on. In addition, these devices should be connected with Internet, or other local network, and accessible to a central web server that organizes and manages the shared model.

There is other advantage of handling virtual digitized labels on a shared workspace. The virtual digitized labels are superior to save the locations and record the working logs. Also, layouting labels with fixed rule or alignment is easier than handling of real paper labels. The digitized labels can be exported in other digital formats such as images and PDF documents. The exported document is suitable for sharing the workspace with other persons or participants.

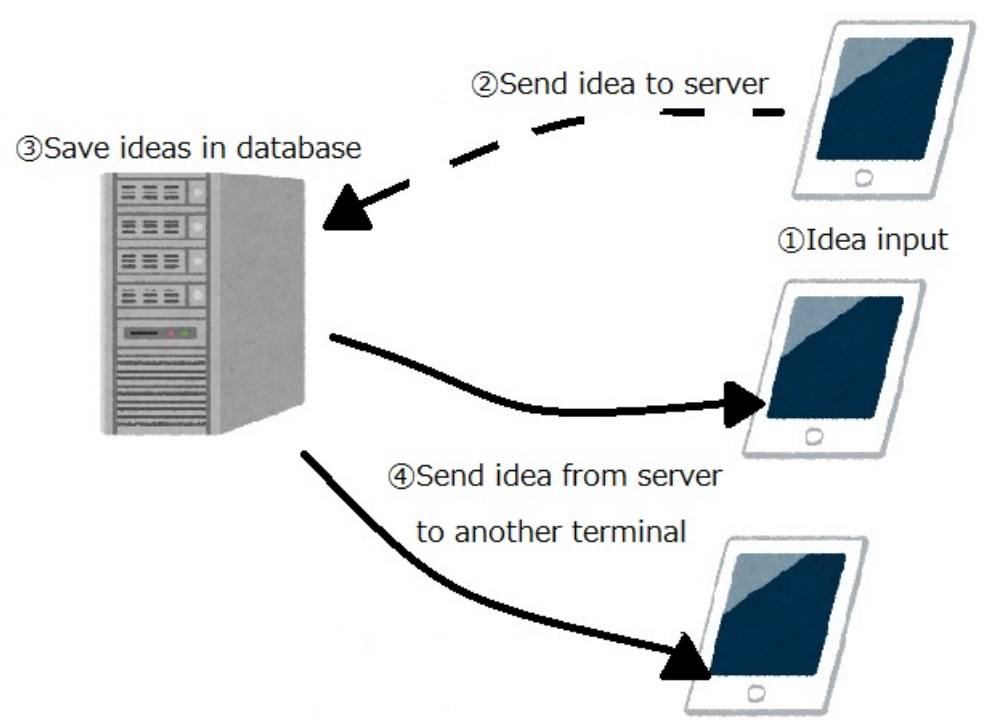

Figure 1: System outline

\section{Implementation}

We adopt Meteor[9, 10] and D3.js[11] to realize shared virtual labels and workspace of group label work especially for KJ method with heterogeneous multi-touch tablets. Figure 1 shows the outline of the proposed system. Meteor is mainly used for serverside imple-mentation of storing/sharing virtual label model, and D3.js visualizes the representation of the label model on clients. 


\subsection{Meteor}

Meteor is a reactive, simple, and powerful application platform based on Node.js written in Javascript. Meteor also incorporates an HTML template feature. Using the feature, the developer can describe web application with simple notations. Since MongoDB database and WebSocket technology are bundled, developers can easily implement an interactive sophisticated web site. Operations in the clients are instantaneously transferred to the server, and stored in the MongoDB database. The modification of the MongoDB database automatically updates the appearance of the client-side. Therefore, Meteor enables automatic synchronization of data among multiple clients. This is beneficial because it is not necessary for the participants to operate updating explicitly. Since the participant do not have to save or update data, they can concentrate on group label work.

\subsection{D3.js}

D3.js[11] is a JavaScript library that is used to draw the dynamic content on the web browser. In an ordinary graph library, all data must be managed and drawn by a program. However, in D3.js, the elements themselves displayed in the graph have data, and it is sufficient to indicate where the elements are placed in the program. Therefore, since the developer can concentrate only on how the data is expressed, complicated visualization becomes possible by D3.js.

\section{Typical Scenario of group label}

In this section, we describe a typical scenario of group label work with the proposed system.

\subsection{Brainstorming}

Figure 2 shows a screenshot of the group label work system. We reproduce labels and workspace of KJ method as a web application of Meteor.

First, participants generate ideas with brainstorming. Each participant uses one tablet to enter his/her ideas. The participant writes out the ideas with the keyboard of the tablet (see Figure 3). The idea label can be added by entering it in the red text input field on the upper side of Figure 3. After that, a label with the idea text is created and placed on the shared workspace. The label created by a participant is immediately shared among the tablets joined to the Meteor server. The participant can specify the color of the label by selecting the drop down list next to the text input field. Since the color of the text input field is changed, the participants can confirm the color of the label.

The buttons 1 to 9 on the lower side of Figure 2 are preset buttons for displaying the screen for each tablet. Figure 4 is a scene of the typical usage with nine tablets. The view of each tablet is determined by the preset button. By considering large virtual space, and each tablet displays a part of the virtual space, the participants can browse and handle many idea labels simultaneously.

Incidentally, the participants can refer the ideas of other participants by pressing a preset button corresponding to another screen. As shown in Figure 5, editing text, copying, and deleting labels can be performed with a pop-up menu. 


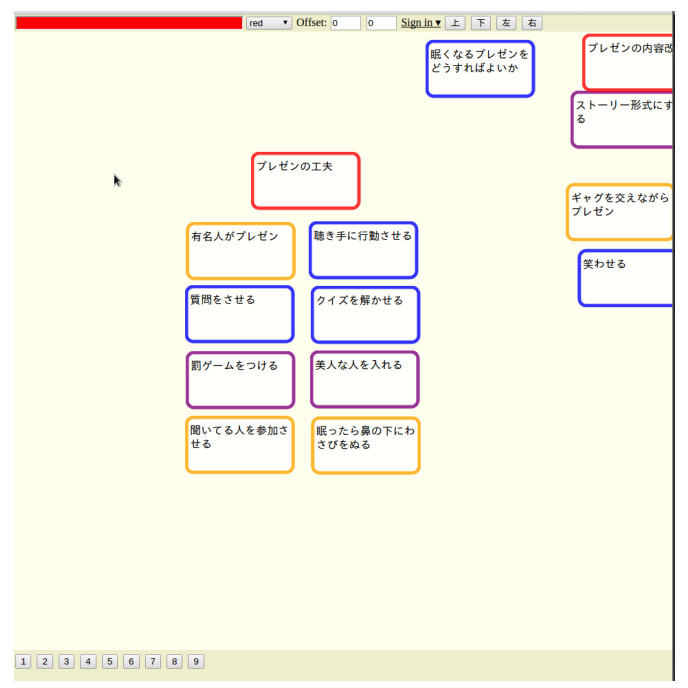

Figure 2: Screenshot of the group label work system

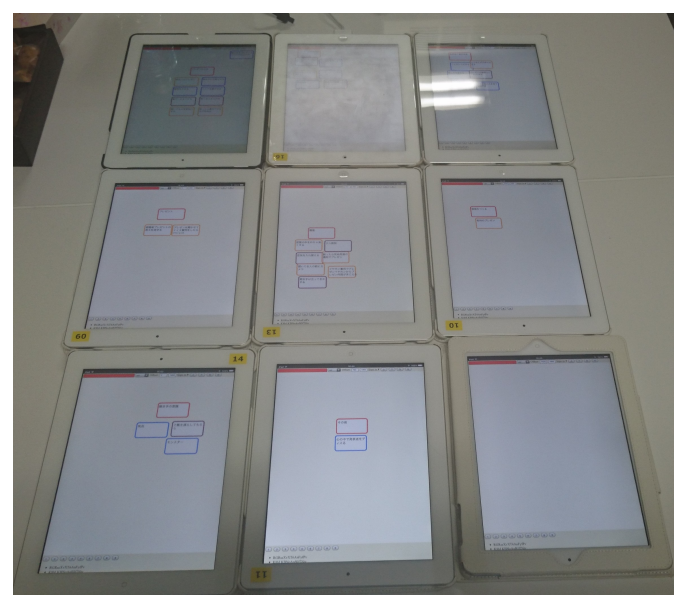

Figure 4: Use of multiple tablet devices

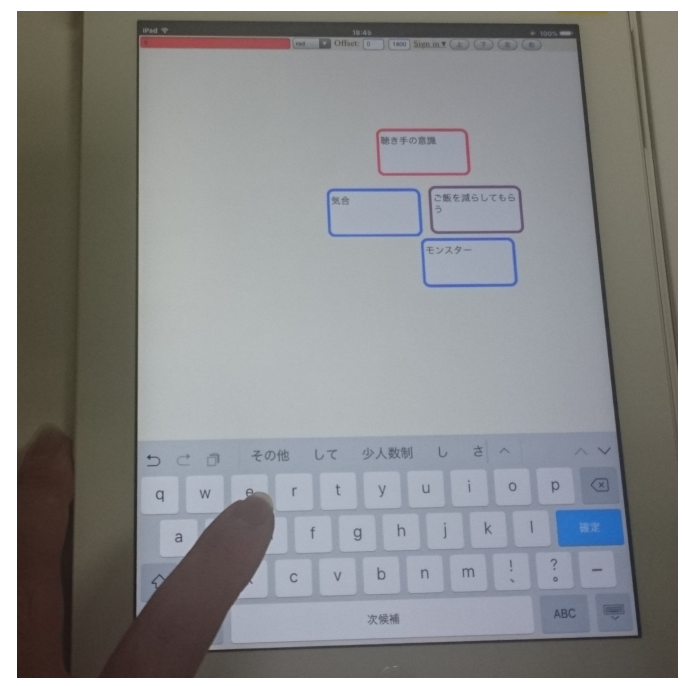

Figure 3: The participant write out an idea in Brainstorming

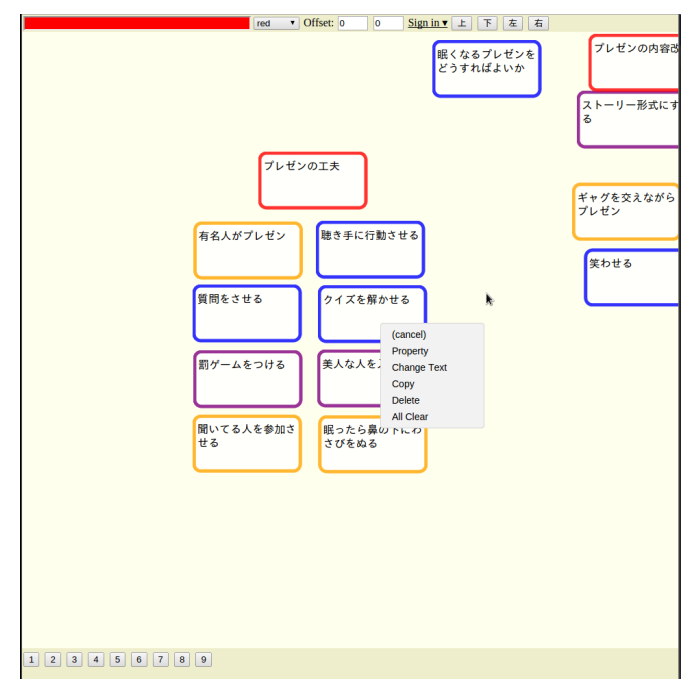

Figure 5: Pop-up Menu of the label 


\subsection{Grouping}

After the brainstorming, the participants move to the grouping phase. In the grouping phase, the participants move labels and collect labels of similar idea as shown in Figure 2, and attach a group title with a red label. As shown in Figure 6, the participants can move the labels by touching and sliding. In this scenario, the participants utilize nine tablets. In such case, the participants gather similar ideas in a screen of tablet. When the participants move the label between tablets, they have to slide in long distance. It is not effective for casual operation. Therefore, we implemented a function of "throwing" by flicking labels. By adopting the throwing, long distance movement of labels are facilitated by the system. For the implementation of the function, we referred a paper of "throwing icon interface" by Kuno et al. [12].

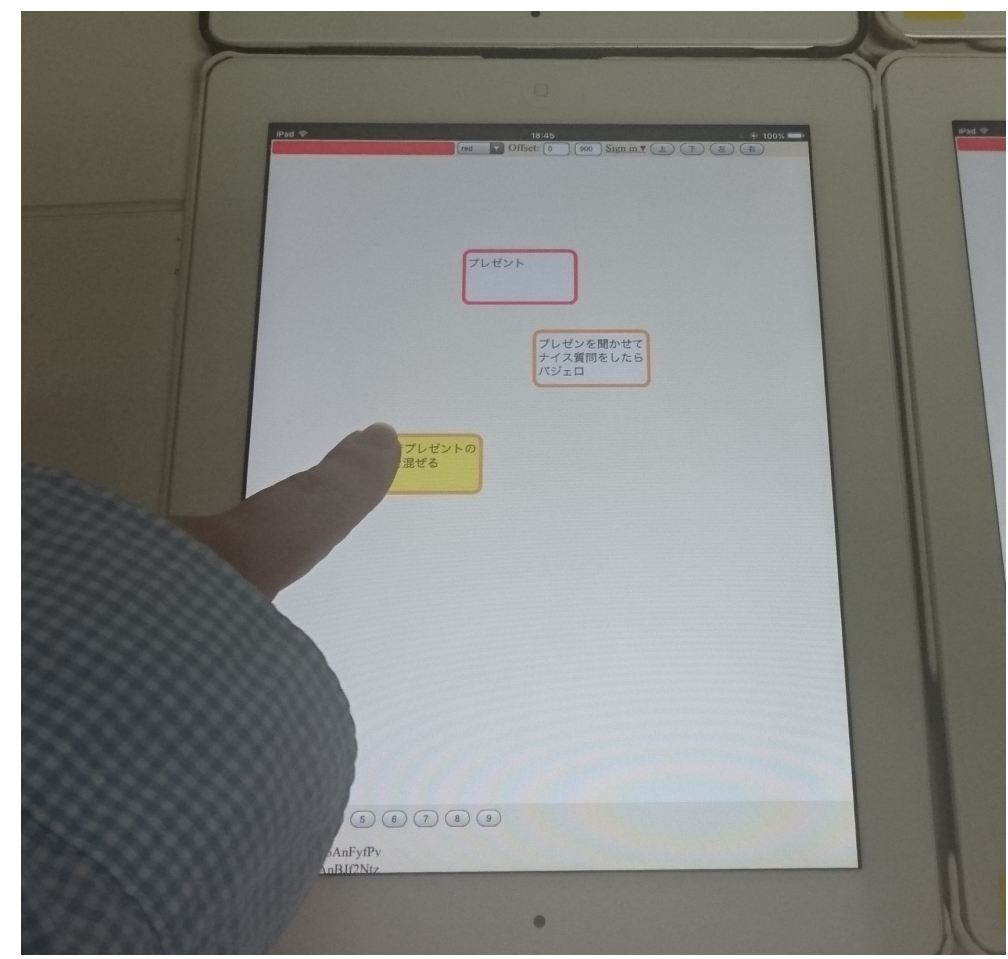

Figure 6: Moving a label by tap-and-slide operation

\section{Related works}

There have been developed many tools and systems that can support creative thinking utilizing virtual card-based activity. KJ Editor [13], GUNGEN [14], and D-ABDUCTOR [15] were tools to support the KJ method by providing a virtual environment. These tools enable users to handle virtual cards on a conventional PC. For multiple PC environment, Yuizono et al. developed a KJ method groupware KUSANAGI[16, 17] based on a multiple PC/mouse middleware GLIA[18]. Since GLIA and KUSANAGI was implemented as a Java program, these systems were platform independent. Gugeek [19] was a web-based multiple user creativity support groupware. Gugeek also employed modern web technologies, but focused 
on the support of distributed PC users. Our system mainly supports co-located sessions with multiple tablets, similar to the paper-based interface.

Kokogawa et al.[20] developed GUNGEN-PHOTO, an idea creation groupware which utilizes photos. GUNGEN-PHOTO has a collaborative workspace and a personal workspace, and there are two main functions which photo extension for effectively extracting ideas from photos and addition of comments from personal workspace. They conducted experiments on idea generation, and found that more ideas were generated when photos were used. For hardwares, GUNGEN-PHOTO employs tablets for providing personal workspace, and a shared projected screen for collaborative workspace. Our system framework utilizes tablets for both the personal and collaborative workspace seamlessly. In addition, our system framework does not require installation of any special software except a web browser.

Ito et al.[21] proposed a tabletop interaction environment that allows users to see a virtual shared workspace extended over a tabletop through the displays of tablet terminals. Physical movement of the tablet terminal intuitively determines the projection of the virtual shared workspace. They also conducted an empirical study of the effectiveness of the "tablet scrolling" interaction by comparing with "swipe-scroll." Their proposed method requires less tablets rather than our system. However, our framework facilitates organizing a tiled large screen by introducing heterogeneous tablet devices.

\section{Experiment}

We conducted an experiment of collaborative group work environment. The purpose of the experiment was to compare the environment of paper-based workspace with that of tablet-based system in efficiency, effectiveness and usability.

For the proposed group label work system, nine tablets (Apple iPad 2) were used. For the conventional method, we used large paper sheet $(788 \mathrm{~mm} \times 1085 \mathrm{~mm})$ and paper label $(38 \mathrm{~mm} \times 63 \mathrm{~mm})$.

The following descriptions are about the common conditions. For each group work session, three participants formed a group. We asked twelve participants in total. Therefore we gathered four groups.

We prepared two themes of group label work, and performed experiments with combinations of themes and methods as shown in Table 1. The theme 1 was "How to relieve negative feelings," and the theme 2 was "How to make a good presentation." A participant group was asked the brainstorming phase first. After that, the participant worked in the grouping phase. The duration of each phase was 15 minutes.

The number of ideas, the number of groups of ideas, and the conversation time were acquired. We also recorded the participants with a video camera. We measured conversation time from the recorded video. After finishing the experiment of both methods, we conducted a questionnaire survey to the participants. The items of the questionnaire survey are shown in Figure 9 and Figure 10. We also gathered comments from participants after the experiment through interviews.

\subsection{Results}

Table 2 and Table 3 show the total number of ideas and the number of groups (islands) for theme 1 and theme 2, respectively. In order to confirm the difference between the themes, we conducted a t-test at the significant level of $5 \%$ for the total number of ideas and the number of groups. The result showed that there were no significant differences between 
Table 1: Combination of theme and method of experiment group

\begin{tabular}{c|c|c}
\hline Group & First & Second \\
\hline \hline A & $\begin{array}{c}\text { Theme 1 } \\
\text { Paper }\end{array}$ & $\begin{array}{c}\text { Theme 2 } \\
\text { Tablet }\end{array}$ \\
\hline B & $\begin{array}{c}\text { Theme 1 } \\
\text { Tablet }\end{array}$ & $\begin{array}{c}\text { Theme 2 } \\
\text { Paper }\end{array}$ \\
\hline C & $\begin{array}{c}\text { Theme 2 } \\
\text { Paper }\end{array}$ & $\begin{array}{c}\text { Theme 1 } \\
\text { Tablet }\end{array}$ \\
\hline D & $\begin{array}{c}\text { Theme 2 } \\
\text { Tablet }\end{array}$ & $\begin{array}{c}\text { Theme 1 } \\
\text { Paper }\end{array}$ \\
\hline
\end{tabular}

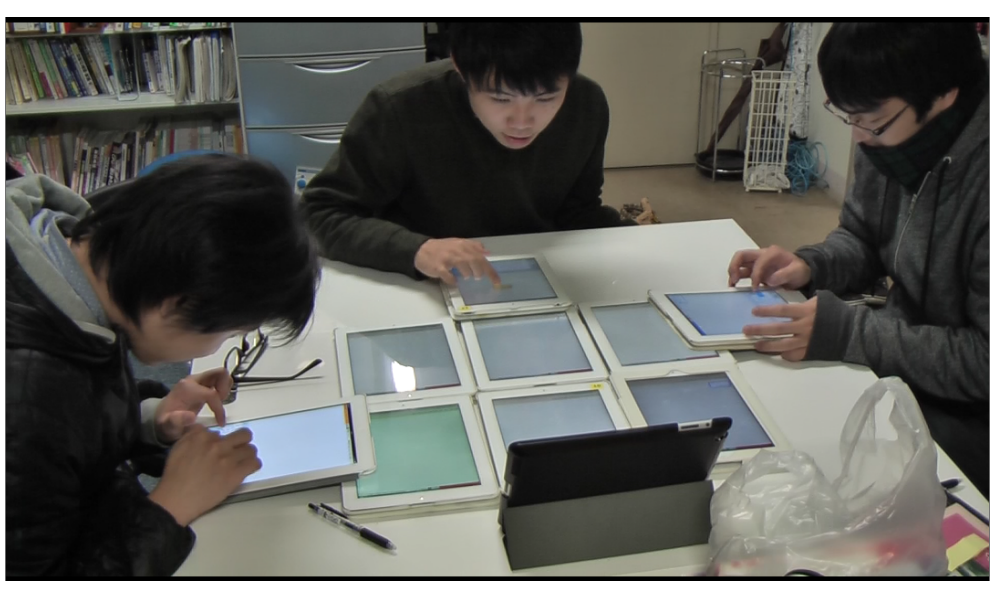

Figure 7: A scene of experiment (grouping phase with tablets)

the themes $(t(3)=2.76, p=.175)$. Therefore, we can proceed the further discussion without the affect of the themes.

Regarding the method, we conducted a t-test for the total numbers of ideas generated at the significant level of $5 \%$ for Table 4 . However, the result showed that there was no significant difference between the method $(t(3)=2.45, p=.081)$.

Figure 8 shows the conversation time during the experiment. Regarding the method, we conducted a t-test for the conversation times. However, the result showed that there was no significant difference between the method in the brainstorming phase $(t(3)=$ $-0.71, p=.785)$ and the grouping phase $(t(3)=-0.69, p=.633)$. 
Table 2: Number of total ideas and groups at theme 1

\begin{tabular}{|c|c|c|}
\hline Group & Total number of ideas & Number of groups (islands) \\
\hline A (paper) & 42 & 10 \\
D (paper) & 50 & 10 \\
B (tablet) & 38 & 8 \\
C (tablet) & 32 & 6 \\
\hline
\end{tabular}

Table 3: Number of total ideas and groups at theme 2

\begin{tabular}{|c|c|c|}
\hline Group & Total number of ideas & Number of groups (islands) \\
\hline B (paper) & 34 & 9 \\
C (paper) & 39 & 5 \\
A (tablet) & 28 & 3 \\
D (tablet) & 34 & 8 \\
\hline
\end{tabular}

Table 4: Total number of ideas

\begin{tabular}{|c|c|c|}
\hline Group & Tablet & Paper \\
\hline A & 28 & 42 \\
B & 38 & 34 \\
C & 32 & 39 \\
D & 34 & 50 \\
\hline
\end{tabular}

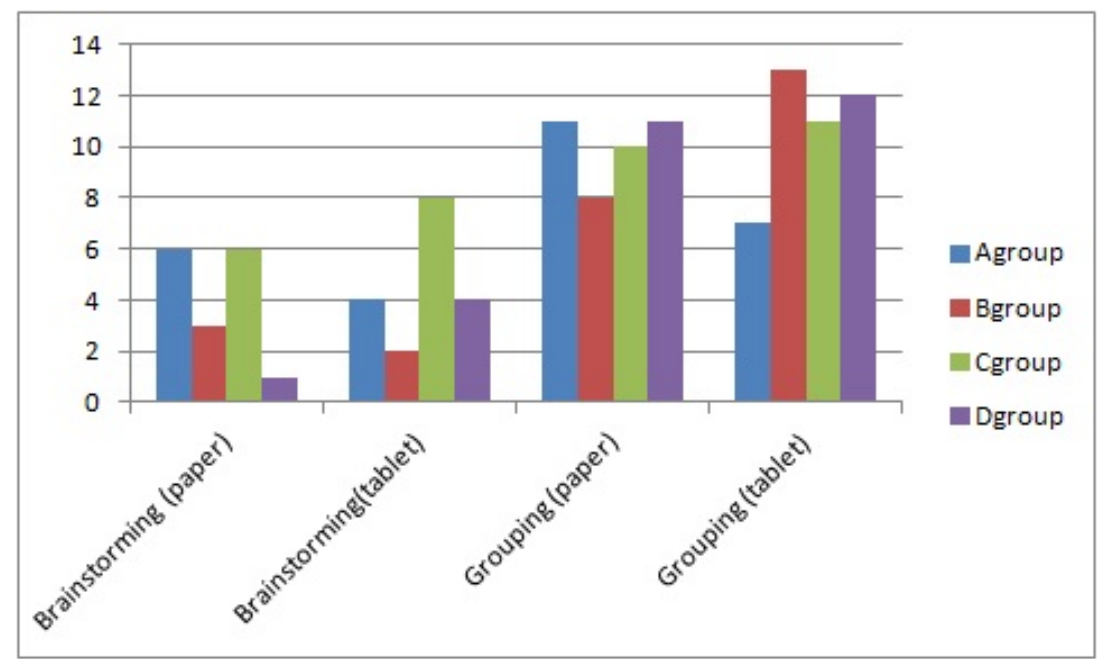

Figure 8: Conversation time (minutes) 


\subsection{Questionnaire survey}

After each experiment of tablet-based proposal method and paper-based conventional method, participants were asked to answer the questionnaire. Figure 9 and Figure 10 show summaries of the questionnaire result. Regarding the summaries, the participants felt difficulty and stress in moving labels on tablets in the grouping phase. By easing the long distance movement, we introduced the throwing by flicking. However, the operation required some techniques, such as holding labels longer time before throwing, and appropriate flicking speed was necessary for expected throwing distance. Due to the less intuitive operation, some participant felt difficulty and stress.

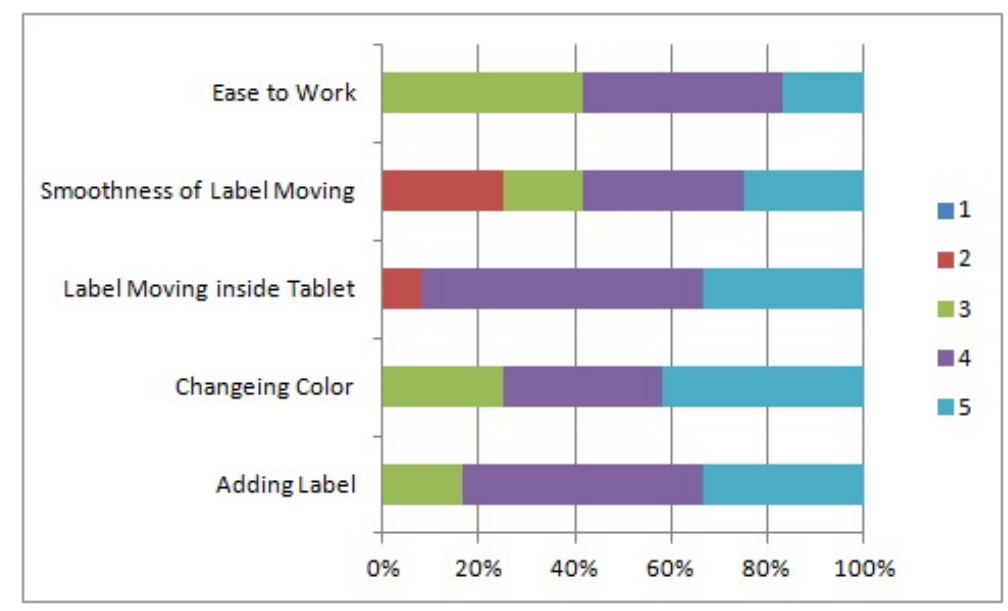

Figure 9: Ease to use during Brainstorming

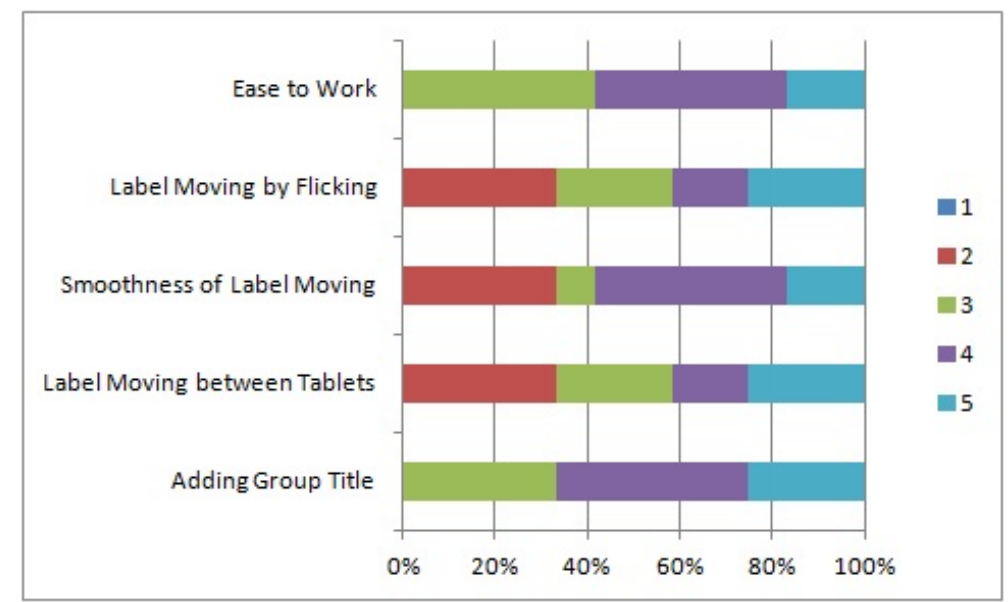

Figure 10: Ease to use during Grouping

\subsection{Discussions}

Regarding the results of the statistics test of the experiments, no significant differences were found. Therefore, we consider that the methods could be chosen by the merits and 
the demerits. For the paper method, fixing labels on the large paper sheet by a glue is troublesome. Also awful handwriting might reduce the effectiveness of sharing ideas during the grouping phase. For the tablet method, the typed text improves the readability of the label text. However, in this implementation, drawing a figure cannot be supported. We will enhance the system to treat handwritten drawing and pictures.

From the interview, we confirmed the positive point of the tablet method. One participant mentioned that it is better for multiple tablets interact with others, and ideas are instantly shared. The instant sharing of ideas could reduce the overlap of the ideas between participants. During the experiment, the number of overlap ideas in the paperbased method seemed larger than that of the tablet-based method. This could be affected by the promotion level of the idea sharing. The overlap itself is not a critical issue to be avoided. In order to generate various ideas in a short time, we have to consider the control of idea sharing.

\section{Conclusion and Future work}

In this paper, we have proposed and implemented a group label work system using multiple tablets, and evaluate the system. In the experiment, we asked participants to perform group label work by both proposed method using tablet and conventional method using paper. Regarding the number of the ideas generated by the participants, t-test revealed that no significant difference was found for the methods. Therefore, the proposed method can be an alternative for the paper-based method.

From the participants' comments through interview, we could obtain a positive feeling in the instant sharing of ideas by multiple tablets. This means that the validity of the proposed approach that realizes real-time updating of idea labels with standard web technologies was confirmed. However, we also received several issues to be improved for further development as the comments. We should improve the editing functions as well as the usability of the system.

\section{Acknowledgment}

The part of this research was supported by the fund of JSPS KAKENHI Grant-in-Aid for Scientific Research (C): Grant Number 15K00485.

\section{References}

[1] Jiro Kawakita Memorial Editorial Board, editor. Exploration of Torushika - trend of thought and the possibility of field science. Hirofumido Shimizu Shobo, August 2012.

[2] Motoki Miura, Taro Sugihara, and Susumu Kunifuji. GKJ : Group KJ Method Support System Utilizing Digital Pens. IEICE transactions on information and systems, 94(3):456-464, March 2011.

[3] Jun Rekimoto. SmartSkin: an infrastructure for freehand manipulation on interactive surfaces. In Proceedings of the SIGCHI conference on Human factors in computing systems, pages 113-120. ACM, 2002. 
[4] Paul Dietz and Darren Leigh. DiamondTouch: a multi-user touch technology. In Proceedings of the 14th annual ACM symposium on User interface software and technology, pages 219-226. ACM, 2001.

[5] Jefferson Y Han. Low-cost multi-touch sensing through frustrated total internal reflection. In Proceedings of the 18th annual ACM symposium on User interface software and technology, pages 115-118. ACM, 2005.

[6] Andrew D Wilson. PlayAnywhere: a compact interactive tabletop projectionvision system. In Proceedings of the 18th annual ACM symposium on User interface software and technology, pages 83-92. ACM, 2005.

[7] Malte Ubl and Eiji Kitamura. Introducing WebSockets: Bringing Sockets to the Web. https://www.html5rocks.com/en/tutorials/websockets/basics/, October 2010.

[8] Victoria Pimentel and Bradford G Nickerson. Communicating and displaying real-time data with websocket. IEEE Internet Computing, 16(4):45-53, 2012.

[9] Meteor: Build Apps with Javascript. https://www.meteor.com/. (Website. Visited: 20 June 2018).

[10]Isaac Strack. Getting Started with Meteor JavaScript Framework. Packt Publishing Ltd, 2012.

[11]D3.js: Data-Driven Documents. https://d3js.org/. (Website. Visited: 20 June 2018).

[12] Yasushi Kuno, Atsuo Oki, Hiroyasu Tsunoda, and Tadashitakashi Kasukawa. "Icon Throwing" User Interface. http://ci.nii.ac.jp/naid/110003743920. (in Japanese).

[13]Hajime Ohiwa, Naohiko Takeda, Kazuhisa Kawai, and Akichika Shiomi. KJ editor: a card-handling tool for creative work support. Knowledge-Based Systems, 10(1):43-50, 1997.

[14]Jun Munemori and Yoji Nagasawa. GUNGEN: groupware for a new idea generation support system. Information and Software Technology, 38(3):213-220, 1996.

[15]Kazuo Misue, Kiyoshi Nitta, Kozo Sugiyama, Takeshi Koshiba, and Robert Inder. Enhancing D-ABDUCTOR Towards a Diagrammatic User Interface Platform. In Proceedings of KES, pages 359-368, 1998.

[16] Takaya Yuizono, Jun Munemori, and Tomohiro Shigenobu. Effects of groupware for an idea generation with a large collaboration interface. IPSJ Journal, 49(7):2574-2588, 2008. (in Japanese).

[17]Takaya Yuizono and Zeying Yu. Text-shared collaboration in second language using groupware for an idea generation. In Advanced Techniques for Knowledge Engineering and Innovative Applications, pages 56-69, 2013.

[18]Takaya Yuizono, Shinichi Nishimura, and Jun Munemori. Development of Middle-ware GLIA for Connective Wide Collaborative Space with Networked I/O Device. In International Conference on Knowledge-Based and Intelligent Information and Engineering Systems, pages 174-180. Springer, 2006. 
[19] Kobkrit Viriyayudhakorn. Creativity assistants and social influences in KJ-method creativity support groupware. $\mathrm{PhD}$ thesis, Japan Advanced Institute of Science and Technology, 2013.

[20] Tomohiro Kokogawa, Yuji Maeda, Takahiro Matsui, Junko Itou, and Jun Munemori. The Effect of Using Photographs in Idea Generation Support System. Information and Media Technologies, 8(3):898-905, 2013.

[21] Naoto Ito, Hideyuki Takada, and Ian Piumarta. Effectiveness of Tabletop Interaction Using Tablet Terminals in a Shared Virtual Workspace. In Takaya Yuizono, Hiroaki Ogata, Ulrich Hoppe, and Julita Vassileva, editors, Collaboration and Technology, pages 98-114, Cham, 2016. Springer International Publishing. 